Please do not remove this page

RMIT

UNIVERSITY

\title{
Non-volatile and volatile memory behaviour in oxygenated amorphous carbon electrochemical metallisation devices
}

Murdoch, Billy; Raeber, Thomas; Barlow, Anders; McCulloch, Dougal; Partridge, James

https://researchrepository.rmit.edu.au/esploro/outputs/9921863400801341/filesAndLinks?institution=61RMIT_INST\&index=null

Murdoch, B., Raeber, T., Barlow, A., McCulloch, D., \& Partridge, J. (2018). Non-volatile and volatile memory behaviour in oxygenated amorphous carbon electrochemical metallisation devices. Applied Physics Letters, 112(24), 1-5. https://doi.org/10.1063/1.5029402

Document Version: Published Version

Published Version: https://doi.org/10.1063/1.5029402

Repository homepage: https://researchrepository.rmit.edu.au (c) 2018 Author(s).

Downloaded On 2023/04/26 23:18:29 +1000 
Thank you for downloading this document from the RMIT Research Repository.

The RMIT Research Repository is an open access database showcasing the research outputs of RMIT University researchers.

RMIT Research Repository: http://researchbank.rmit.edu.aul

\section{Citation:}

Murdoch, B, Raeber, T, Barlow, A, McCulloch, D and Partridge, J 2018, 'Non-volatile and volatile memory behaviour in oxygenated amorphous carbon electrochemical metallisation devices', Applied Physics Letters, vol. 112, no. 24, pp. 1-5.

See this record in the RMIT Research Repository at:

https://researchbank.rmit.edu.au/view/rmit:49089

Version: Published Version

\section{Copyright Statement:}

(C) 2018 Author(s).

\section{Link to Published Version:}

https://dx.doi.org/10.1063/1.5029402 


\section{Non-volatile and volatile memory behaviour in oxygenated amorphous carbon electrochemical metallisation devices}

Cite as: Appl. Phys. Lett. 112, 242903 (2018); https://doi.org/10.1063/1.5029402

Submitted: 14 March 2018 . Accepted: 30 May 2018. Published Online: 11 June 2018

B. J. Murdoch, T. J. Raeber, A. J. Barlow (D, D. G. McCulloch, and J. G. Partridge

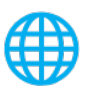

\section{ARTICLES YOU MAY BE INTERESTED IN}

Artificial electronic synapse characteristics of a $\mathrm{Ta} / \mathrm{Ta}_{2} \mathrm{O}_{5-\mathrm{x}} / \mathrm{Al}_{2} \mathrm{O}_{3} / \mathrm{InGaZnO} \mathrm{In}_{4}$ memristor device on flexible stainless steel substrate

Applied Physics Letters 113, 013503 (2018); https://doi.org/10.1063/1.5027776

Non-volatile resistive switching in CuBi-based conductive bridge random access memory device

Applied Physics Letters 112, 253503 (2018); https://doi.org/10.1063/1.5030765

Percolation theory based statistical resistance model for resistive random access memory Applied Physics Letters 112, 253505 (2018); https://doi.org/10.1063/1.5023196

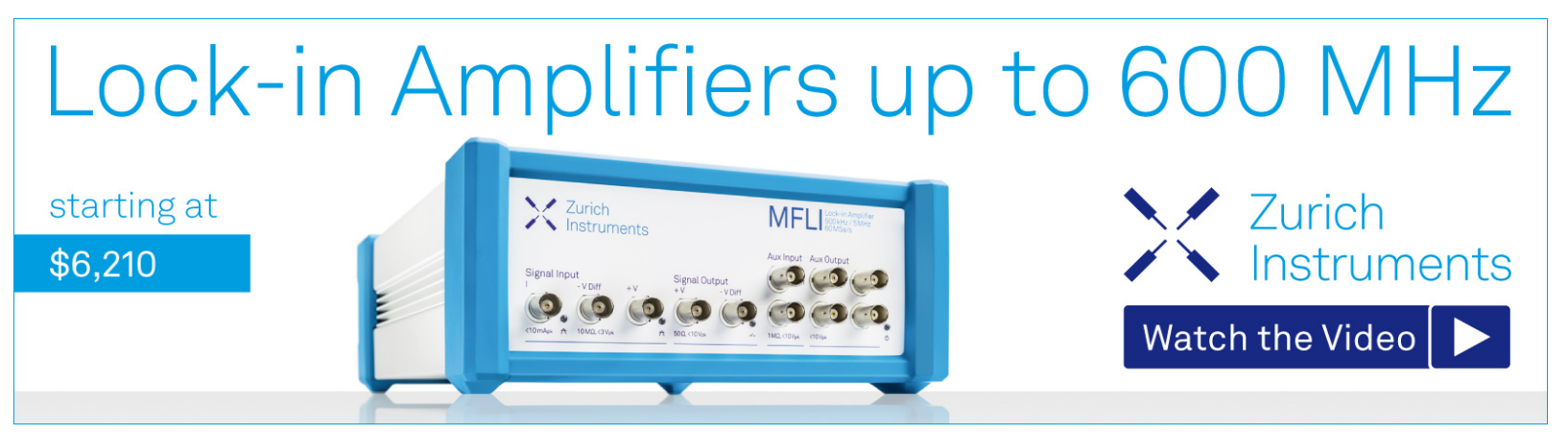




\title{
Non-volatile and volatile memory behaviour in oxygenated amorphous carbon electrochemical metallisation devices
}

\author{
B. J. Murdoch, ${ }^{1, \text { a) }}$ T. J. Raeber, ${ }^{1}$ A. J. Barlow, ${ }^{2}$ D. G. McCulloch, ${ }^{1}$ and J. G. Partridge ${ }^{1}$ \\ ${ }^{1}$ School of Science, RMIT University, VIC 3001 Melbourne, Australia \\ ${ }^{2}$ Centre for Materials and Surface Science (CMSS), Department of Chemistry and Physics, \\ La Trobe University, VIC 3086 Melbourne, Australia
}

(Received 14 March 2018; accepted 30 May 2018; published online 11 June 2018)

\begin{abstract}
The resistive switching behaviour of oxygenated amorphous carbon electrochemical metallisation devices is investigated. The effect of temperature on the microstructure and composition of the oxygenated carbon matrix is also investigated by annealing in situ in a transmission electron microscope. The devices exhibit controllable bipolar non-volatile and bi-directional volatile resistive switching behaviour that is dependent on the resistance state of the device and the polarity of the RESET voltage. The characteristics presented suggest suitability for incorporation into neuromorphic computing and memory storage technologies as memory cells, selector devices, or synaptic emulators. Published by AIP Publishing. https://doi.org/10.1063/1.5029402
\end{abstract}

Resistive random access memory (RRAM) devices and related memristor devices are the subject of an intensive research effort due to their suitability for non-volatile data storage and cognitive computing. ${ }^{1,2}$ While metal oxide-based resistive switches comprise the largest sub-class of these devices, carbon-based devices, and amorphous carbon-based memory devices in particular, have recently been recognised by their addition to the International Technology Roadmap for Semiconductors (ITRS). ${ }^{3}$

There are three main approaches to producing nonvolatile two-terminal amorphous carbon memory. (1) Resistive switching in tetrahedral amorphous carbon (ta-C), ${ }^{4-6}$ which can be switched to a low resistance state (LRS) from a high resistance state (HRS) via thermally induced clustering of $s p^{2}$ bonds to form networks of conductive $s p^{2}$ clusters. Reversal from HRS to LRS is then achieved by applying short pulses of high instantaneous power designed to heat and quench the $s p^{2}$ clusters such that a fraction of the bonds revert to $s p^{3}$. (2) Oxygenated amorphous carbon $\left(\mathrm{a}-\mathrm{CO}_{\mathrm{x}}\right) \mathrm{RRAM}^{7-9}$ which operates based on the electrochemical redox reaction of a-C and oxygen, and (3) electrochemical metallisation (ECM) memory cells that work based on electric field-induced drift of metal ions from an active electrode through an a-C matrix to form conducting filaments $(\mathrm{CFs}){ }^{10-12}$ The $\mathrm{CFs}$ can be ruptured upon the application of reverse bias.

ECM memory is unique among the different classes of a-C resistive switching devices because both non-volatile and volatile switching can be observed. The dynamic volatile switching is enabled by the diffusion (rather than drift) of metal ions within the matrix. It has been reported that the type of switching observed (volatile/non-volatile) can be actively controlled by limiting the growth of the $\mathrm{CF} .{ }^{11}$ That is to say, a CF of larger diameter will exhibit non-volatile resistive switching, while a $\mathrm{CF}$ of smaller diameter may be ruptured by thermodynamic relaxation after the cessation of bias application. Devices with these types of diffusive dynamics are of particular interest in neuromorphic computing for the

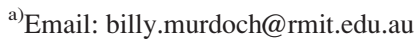

emulation of $\mathrm{Ca}^{2+}$ dynamics in chemical synapses. ${ }^{13}$ They may also function as selector devices in crossbar circuits. ${ }^{14}$

A recent study on carbon-based ECMs has also highlighted the importance of Joule-heating induced $s p^{2}$ clustering of $\mathrm{C}$ atoms for tailoring the $\mathrm{CF}$ size and uniformity. ${ }^{12}$ Metal ion drift under a uniform applied bias can result in large fluctuations in the switching behaviour of devices. ${ }^{12}$ To address this problem, it was demonstrated that filaments composed of $s p^{2}$ clusters guide the metal ions by locally enhancing the electric field and in-turn reducing the stochastic creation of multiple metal CFs. Additionally, it would be expected that, as diffusivity is blocked by ta-C, Joule heating-induced production of $s p^{2}$ clusters will help metal ions to become itinerant. ${ }^{15}$ Thus, the understanding and control of the microstructure of a-C in ECM memory during operation is vital.

Here, we report on the fabrication and characterisation of a- $\mathrm{CO}_{\mathrm{x}}$ ECM devices. The devices exhibit controllable bipolar non-volatile and bi-directional volatile resistive switching behaviour that is dependent on the resistance state of the device and the polarity of the RESET voltage. The effect of temperature on the microstructure and composition of the $\mathrm{a}-\mathrm{CO}_{\mathrm{x}}$ matrix has been revealed by annealing in situ in a transmission electron microscope (TEM) and ex situ Raman spectroscopy.

A structural diagram of the ECM cell is shown in Fig. 1(a). A $20 \mathrm{~nm}$ thin film of $\mathrm{Ag}$ was deposited onto a $\mathrm{Si}$ wafer with a $1 \mu \mathrm{m}$ thermally grown oxide insulator. A $16 \mathrm{~nm}$ thick a- $\mathrm{CO}_{\mathrm{x}}$ layer was then deposited through a carbon-coated mesh grid at $-100 \mathrm{~V}$ bias by filtered cathodic vacuum arc (FCVA) deposition in a $0.5 \mathrm{mTorr}^{\mathrm{O}_{2}}$ atmosphere. The substrate temperature during deposition was $100{ }^{\circ} \mathrm{C}$. Lithographically defined circular Pt contacts of diameter $100 \mu \mathrm{m}^{2}$ were sputter-deposited.

Figure 1(b) shows several current-voltage $(I / V)$ curves for the $\mathrm{a}-\mathrm{CO}_{\mathrm{x}} \mathrm{ECM}$ devices. A "forming" sweep was required as a pre-step prior to operation. During this process, the compliance current was set to $1 \mathrm{~mA}$ to prevent the device from permanently shorting. This is required due to the capacitive discharge current, which can cause a current overshoot on the order of $10 \mathrm{~mA} .^{16}$ Typical non-volatile resistive 
(a)
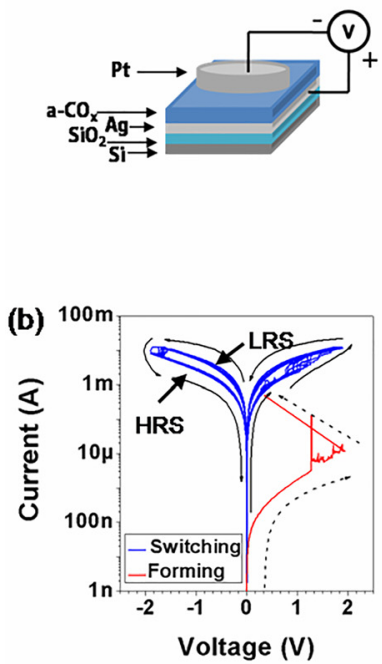

(c)

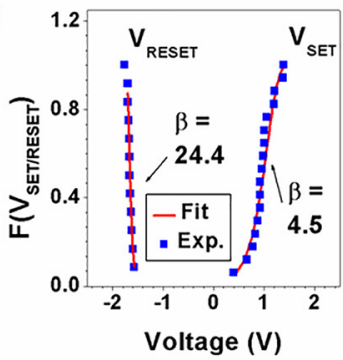

(d)

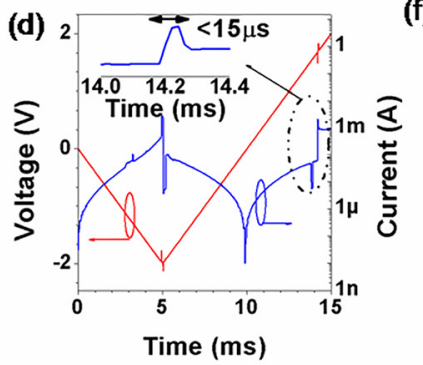

(e)

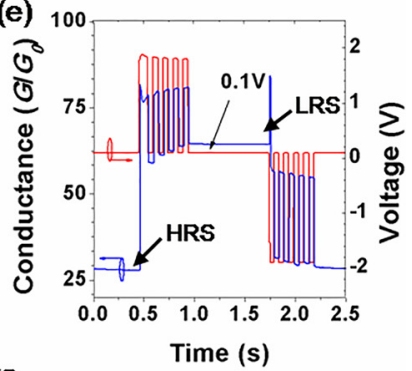

(f)

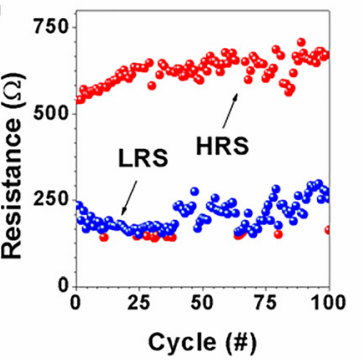

FIG. 1. (a) Structural diagram of a-CO memory devices. (b) Typical $I / V$ curves during the "forming" and switching processes. (c) Cumulative probability distribution of the SET and RESET voltages. (d) Time-dependence of typical I/ $\mathrm{V}$ curves. The inset shows the switching speed of the SET function. (e) Device response to potentiating and depressing programming pulses. (f) Distribution of resistance states of the device in response to repeated cycling of the programming pulses in (e). switching behaviour is observed. There is a distribution of SET voltages $\left(\mathrm{V}_{\mathrm{SET}}\right)$ in the forward bias range. However, the RESET voltage $\left(\mathrm{V}_{\mathrm{RESET}}\right)$ remains relatively uniform. Figure 1(c) shows the cumulative probability distributions of $\mathrm{V}_{\text {SET }}$ and $\mathrm{V}_{\text {RESET }}$. These distributions were analysed using Weibull statistics ${ }^{17}$

$$
F(x)=1-\exp \left[\left(-\frac{x}{x_{0}}\right)^{\beta}\right],
$$

where $F$ is the probability of a SET/RESET occurring, $x$ is $\mathrm{V}_{\mathrm{SET} / \mathrm{RESET}}, x_{0}$ is the maximum value of $\mathrm{V}_{\mathrm{SET} / \mathrm{RESET}}$, and $\beta$ is the Weibull slope (or shape factor), which is a measure of the dispersion of SET and RESET conditions. The distributions were characterised by a single $\beta$, indicating that switching occurs via a single $\mathrm{CF} .{ }^{18}$ Figure $1(\mathrm{~d})$ shows the switching speed of the SET function during a $I / V$ sweep. The switching speed of the SET and RESET functions was $<15 \mu$ s. Figure 1(e) shows the device response to a series of 5 potentiating voltage pulses $(1.9 \mathrm{~V}, 50 \mathrm{~ms}$ duration) followed by 5 pulses of identical magnitude and duration of the opposite polarity (depression). The device conductance between pulses was measured with a non-perturbative voltage of $0.1 \mathrm{~V}$. Figure 1(e) shows the distribution of the HRS and LRS over 100 repetitions of the programming sequences shown in Fig. 1(f). Note that the maximum number of non-volatile switches for a device was $>10^{3}$ cycles. Both the HRS and LRS memory states were retained for $>10^{5}$ seconds.

Figure 2(a) shows the electrical transport properties of the $\mathrm{Ag} / \mathrm{a}-\mathrm{CO}_{\mathrm{x}} / \mathrm{Pt}$ device in reverse bias. The HRS $I-V$ curve is shown on a Poole-Frenkel (P-F) ${ }^{19}$ plot in Fig. 2(b). A linear relationship exists between $\operatorname{Ln}(I / V)$ and $\mathrm{V}^{1 / 2}$ over the majority of the voltage range. The P-F conduction model is often used to characterise conduction in a-C. ${ }^{5,20}$ Figure 2(c) shows a linear fit to the $I-V$ characteristics of the LRS and confirms an Ohmic transport mechanism consistent with a metallic CF conduction model. These transport properties and the bipolar non-volatile switching behaviour observed in Fig. 1 support an ECM switching model where $\mathrm{Ag}^{+}$ions form a conducting metal filament by drift towards the Pt anode, induced by the applied electric field. Upon application of reverse bias, the $\mathrm{Ag}^{+}$ions drift back towards the $\mathrm{Ag}$ electrode, rupturing the filament. The conduction in the HRS is facilitated by hopping transport through the a-C matrix. This model is further supported by temperature-dependent conductance measurements of the device in the LRS [Fig. 2(d)]. A positive temperature coefficient of resistance, $\alpha=\sim 2 \times 10^{-3} \mathrm{~K}^{-1}$, agrees with previous observations for Ag nanowires. ${ }^{21}$ The discrepancy between this value and that of high purity bulk $\mathrm{Ag}\left(3.8 \times 10^{-3} \mathrm{~K}^{-1}\right)$ may be caused by scattering from defects in the filament which reduce the electron mean free path.

Unipolar switching is observed in the devices when a forward bias is applied in the LRS. This drives the device to a higher resistance than observed during bipolar switching. Continual voltage sweeping thereafter results in bi-directional threshold switching behaviour, which is shown in Fig. 3(a). Due to the change in switching characteristics, we refer to this as a "2nd forming" step. Note that the threshold switching observed is achieved without lowering the compliance current, in contrast with previous reports. ${ }^{11}$ The SET threshold
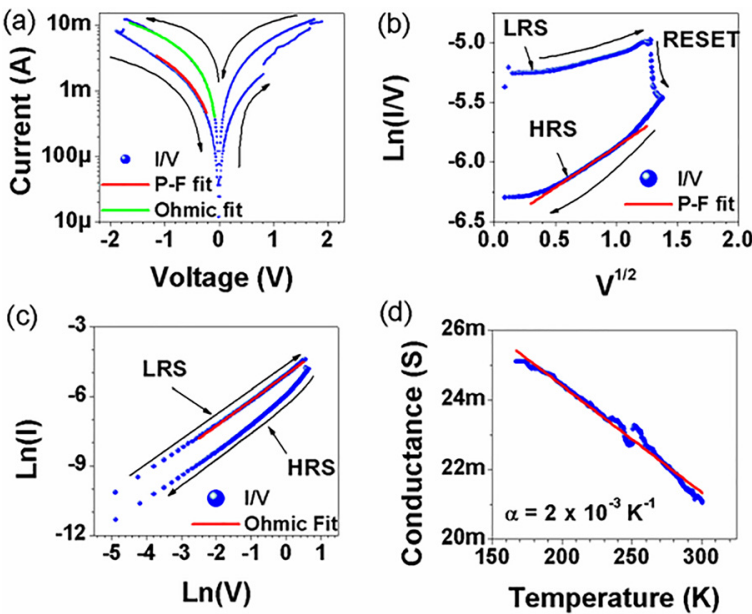

FIG. 2. (a) Electrical transport properties in the non-volatile resistive switching regime. Note that the reverse bias $I / V$ characteristics are considered due to variability in the SET voltage. (b) Poole-Frenkel (P-F) plot in the HRS. (c) Linear fit to $I / V$ properties in the LRS revealing Ohmic conduction. (d) Temperature dependence of the conductance in the LRS. 

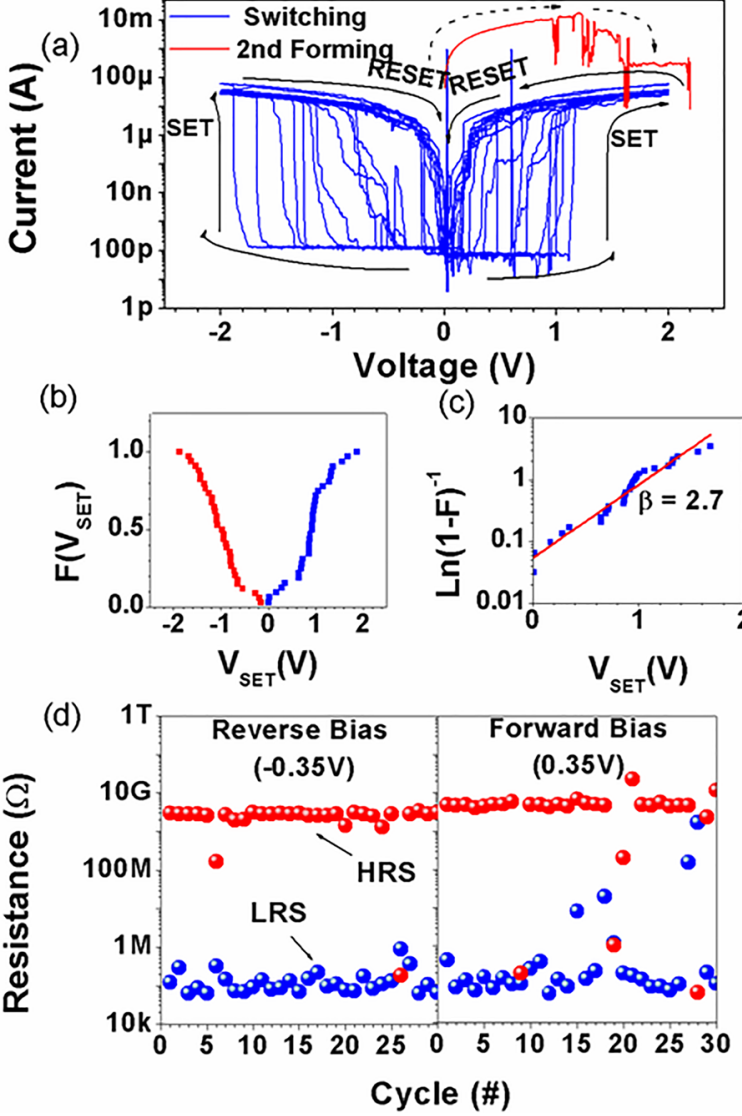

FIG. 3. (a) Repeatable, bi-directional threshold switching after application of a forward bias "2nd forming" step. (b) Cumulative probability distribution of the SET voltages. (c) Weibull statistics of the forward bias $\mathrm{V}_{\text {SET }}$ voltages. The characteristics can be fit to a single slope, revealing the Weibull shape factor, $\beta$. (d) Distribution of HRS/LRS during threshold switching in both forward and reverse bias.

switching voltages are more stochastic than in Fig. 1, while the RESET voltages are, once again, uniform. Figure 3(b) shows the cumulative probability distribution of $\mathrm{V}_{\mathrm{SET}}$ in both bias directions. These were analysed using Weibull statistics [Fig. 3(c)]. The extracted Weibull shape factor $(\beta)$ was determined to be 2.7, indicating a much higher dispersion of $\mathrm{V}_{\mathrm{SET}}$ for volatile resistive switching compared to the non-volatile action [Fig. 1(f)]. The mean $\mathrm{V}_{\mathrm{SET}}$ was determined to be $1.0 \pm 0.4 \mathrm{~V}$ in either bias direction, reflecting symmetry in the switching characteristics. The high and low resistance states measured at a magnitude of $0.35 \mathrm{~V}$ in both forward and reverse bias directions show relatively stable distribution of states over multiple cycles [Fig. 3(d)]; however, a small percentage of the cycles do show non-conformity. This is representative of the non-zero $\mathrm{F}\left(\mathrm{V}_{\mathrm{SET}}\right)$ at low voltages. The on/off ratio was observed to be $>10^{5}$. The high on/off ratio, HRS, and low $\mathrm{V}_{\mathrm{SET}}$ in these device characteristics suggest suitability as selectors for low power memory cells.

In regard to Joule heating effects and the microstructure of the a- $\mathrm{CO}_{\mathrm{x}}$ matrix, it has been noted in previous studies on a-C that Joule heating can cause reordering of $s p^{2}$ bonds to form larger $s p^{2}$ clusters; however, the rehybridisation of $s p^{3}$ bonds to $s p^{2}$ bonds requires temperatures that are higher than the operating temperatures of typical ECM devices $\left(>1100{ }^{\circ} \mathrm{C}\right) .{ }^{12,22,23} \mathrm{We}$ are able to estimate the maximum localised temperature increase in the filament during (non-volatile) RESET by solving the steady-state Fourier heat flow equation ${ }^{22}$

$$
\Delta T=\frac{V_{R E S E T}^{2}}{8 \kappa \rho_{L R S}},
$$

where $V_{\mathrm{RESET}}$ is the reset voltage, $\kappa$ is the thermal conductivity of a $\mathrm{Ag}$ nanowire, and $\rho_{\mathrm{LRS}}$ is the device resistivity in the LRS. The thermal conductivity of a Ag nanowire has been estimated to be $\sim 300-400 \mathrm{~W} \mathrm{~m}^{-1} \mathrm{~K}^{-1}$, whereas the thermal conductivity of a-C is $s p^{2} \%$-dependent but estimated to be $<1 \mathrm{~W} \mathrm{~m}^{-1} \mathrm{~K}^{-1}$, making it negligible. ${ }^{24,25}$ Hence, the maximum temperature of the filament during operation is estimated to be $<700 \mathrm{~K}$.

Figure 4(a) shows the Raman spectra for a- $\mathrm{CO}_{x}$ layers as a function of annealing temperature. The Raman spectra were acquired using a Horiba Jobin Yvon HR800 UV and $532 \mathrm{~nm}$ laser at $1.6 \mathrm{~mW}$. A broad asymmetric band is observed that is related to the $s p^{2}$ components of the a-CO films and can be resolved into the D peak $\left(1355 \mathrm{~cm}^{-1}\right)$ and $\mathrm{G}$ peaks $\left(1500-1600 \mathrm{~cm}^{-1}\right)$. The D peak intensity increases relative to the $\mathrm{G}$ peak as the annealing temperature increases, as shown in Fig. 4(b). This ratio is related to the diameter of the $s p^{2}$ clusters, $L_{a}$, by

$$
\frac{I(D)}{I(G)}=\frac{C(\lambda)}{L_{a}}
$$

where $C(\lambda)$ is $\approx 0.0055 .^{26}$ The rise in $I(\mathrm{D}) / I(\mathrm{G})$ and $L_{a}$ observed in Fig. 4(b) after annealing at temperatures up to $673 \mathrm{~K}$ demonstrates that clustering of $s p^{2}$ bonds occurs within the estimated temperature range covered by the a- $\mathrm{CO}_{\mathrm{x}}$ memory devices.

(a)
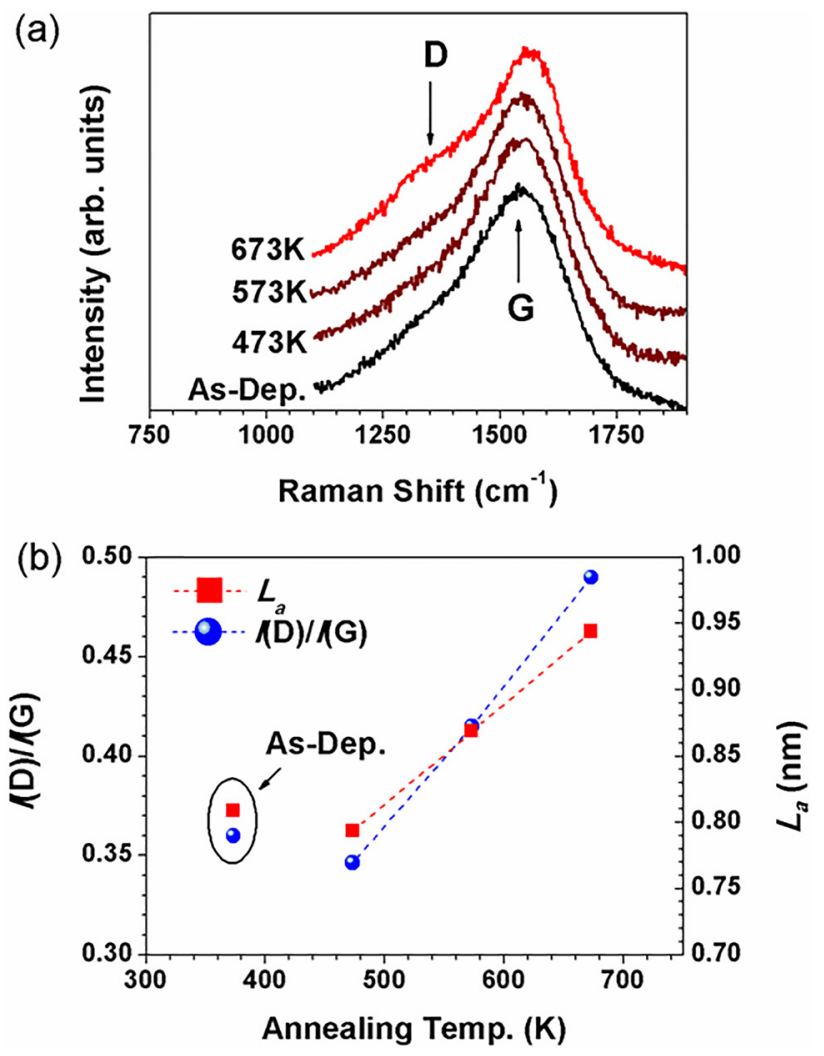

FIG. 4. (a) Raman spectra for a- $\mathrm{CO}_{\mathrm{x}}$ layers annealed in vacuo. (b) $I(\mathrm{D}) / I(\mathrm{G})$ peak intensity ratio and $s p^{2}$ cluster diameter as a function of annealing temperature. 
To further investigate the effect of temperature on the microstructure and composition of the a- $\mathrm{CO}_{\mathrm{x}}$ films, we performed electron energy loss spectroscopy (EELS) and energy dispersive X-ray spectroscopy (EDS) inside a TEM (JEOL 2100F equipped with a Gatan imaging filter). Samples were annealed in situ. To achieve this, an a- $\mathrm{CO}_{\mathrm{x}}$ thin film was deposited onto a commercially available in situ heating chip (Protochips Fusion Model. E-FHBS) with an electron transparent $\mathrm{Si}_{3} \mathrm{~N}_{4}$ membrane window. The samples were held at each temperature for $5 \mathrm{~min}$ and then cooled to $300 \mathrm{~K}$ before analysis. Figure 5(a) shows the C K edge EELS as a function of annealing temperature for $\mathrm{a}-\mathrm{CO}_{\mathrm{x}}$ thin films. The main features are the $1 s \rightarrow \pi^{*}$ peak, arising predominantly from C-C $s p^{2}$ bonding, and the $1 s \rightarrow \sigma^{*}$ states. Peaks relating to C-O bonding are not clearly resolvable in most of the spectra but occur at $287-288 \mathrm{eV} .{ }^{27}$ As the sample was annealed, the $\pi^{*}$ peak became sharper and the $\sigma^{*}$ peak became flatter, indicative of graphitisation. The $\mathrm{C}-\mathrm{O}$ bonding prior to annealing is revealed more clearly by room-temperature $\mathrm{X}$-ray photoelectron spectroscopy (XPS) (obtained using a Kratos Axis Nova). Figure 5(b) shows the C $1 s$ XPS spectrum and reveals that the dominant carbon-to-oxygen bonding in the bulk a- $\mathrm{CO}_{\mathrm{x}}$ film was singly bonded $\mathrm{C}-\mathrm{O}-\mathrm{C} / \mathrm{C}-\mathrm{O}-\mathrm{H}$, with a small amount of $\mathrm{C}=\mathrm{O}-\mathrm{C}$. Figure $5(\mathrm{c})$ shows the $s p^{2} \%$ derived from the $\mathrm{C} \mathrm{K}$ edge EELS as a function of temperature. The asdeposited film showed a high percentage of $s p^{2}$ bonds, which is attributed to hydrocarbon contamination that was volatised and removed from the sample after annealing. An increase in the $\mathrm{C}-\mathrm{C} s p^{2}$ bonding in the sample was observed beginning at a temperature of $\sim 873 \mathrm{~K}$. This is a lower temperature than expected for a-C but is similar to that for amorphous carbonbased compounds, such as $\mathrm{CN}_{\mathrm{x}}{ }^{28}$ Nevertheless, this onset temperature exceeds the operational limit of the $\mathrm{Ag} / \mathrm{a}-\mathrm{CO}_{\mathrm{x}} / \mathrm{Pt}$ device and, hence, the bulk $s p^{2}$ fraction is not expected to change dramatically as a function of Joule-heating. However, the $s p^{2} \%$ may still be reduced locally via oxidation. The effect of temperature on the RESET of oxygenated amorphous carbon memristors has previously been investigated, and it was determined that RESET functions could be induced by the redistribution of oxygen during heating to
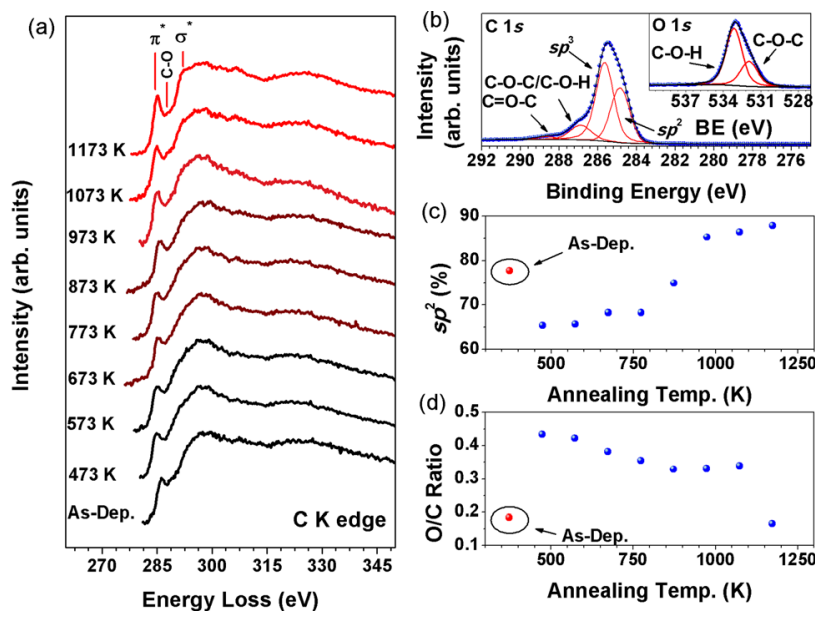

FIG. 5. (a) $\mathrm{C} \mathrm{K}$ edge EELS for a-CO $\mathrm{CO}_{\mathrm{x}}$ annealed in situ. (b) $\mathrm{C} 1 \mathrm{~s}$ XPS spectrum $\left(\mathrm{O} 1 s\right.$ is shown in the inset) for as-deposited a-CO $\mathrm{CO}_{\mathrm{x}}$. (c) $s p^{2} \%$ as a function of vacuum annealing temperature as determined by core-loss EELS. (d) $\mathrm{O} / \mathrm{C}$ ratio determined by EDS.
550-600 K. ${ }^{8}$ Figure $5(\mathrm{~d})$ shows the $\mathrm{C} / \mathrm{O}$ ratio measured by EDS. Loosely bound oxygen, likely to be hydroxyls that were observed in the O $1 s$ XPS spectrum [inset Fig. 5(b)], is removed beginning at temperatures $>573 \mathrm{~K}$. Approximately $20 \%$ of the total oxygen is removed before the rate plateaus at $\sim 873 \mathrm{~K}$. At temperatures $>1073 \mathrm{~K}$, the oxygen content decreases sharply as the film graphitises. Therefore, the majority of the oxygen in the bulk is not expected to diffuse due to the temperature rise near the filament alone. These results show that limited oxygen diffusion occurs due to local heating at the filament and the application of an electric field is required to mobilise oxygen in the device.

We now turn our attention to the threshold switching mechanism, which is illustrated in Fig. 6. Previous work has shown that threshold switching in a-C ECM devices depends on the $s p^{2}$ cluster size. ${ }^{11,12}$ In our devices, the current is limited by performing the 2 nd forming process in which we believe that electrochemical oxidation of the filament (including the $s p^{2}$ clusters) takes place [Fig. 6(b)]. This assertion is made because the 2 nd forming process has not been observed in reverse bias. Previously, unipolar switching has been explained in metal oxides $\left(\mathrm{TiO}_{2}\right.$ for example) by a conical filament growth model. ${ }^{29,30}$ The RESET mechanism was ascribed to the passivation of oxygen vacancies by thermally activated migration of oxygen ions. This is useful in explaining the 2 nd forming process observed in our a- $\mathrm{CO}_{\mathrm{x}}$ devices. The filament temperature during $2 \mathrm{nd}$ forming is estimated to be $550-600 \mathrm{~K}$, the temperature at which loosely bound oxygen mobilises in the matrix [see Fig. 5(d)]. However, high current alone is not sufficient to induce the 2nd forming step because this temperature is lower than during RESET functions in reverse bias, so the switching is unlikely to be an entirely thermally driven process. In forward bias, $\mathrm{O}^{-}$ions (sourced from the a-CO $\mathrm{CO}_{\mathrm{x}}$ matrix) are attracted towards the positive Ag electrode and positively biased $\mathrm{CF}$, which reduces its diameter and the size of the $s p^{2}$ clusters via oxidation. The stochastic threshold switching behaviour observed thereafter is consistent with that of a-C devices operated with low compliance currents to limit their CF diameter. ${ }^{11,12}$

The conductance during the non-volatile resistive switching is $\gg 2 e^{2} / h$, which for a metallic CF in an ECM cell means that a continuous filament is formed due to cation accumulation induced along the conductive path by the

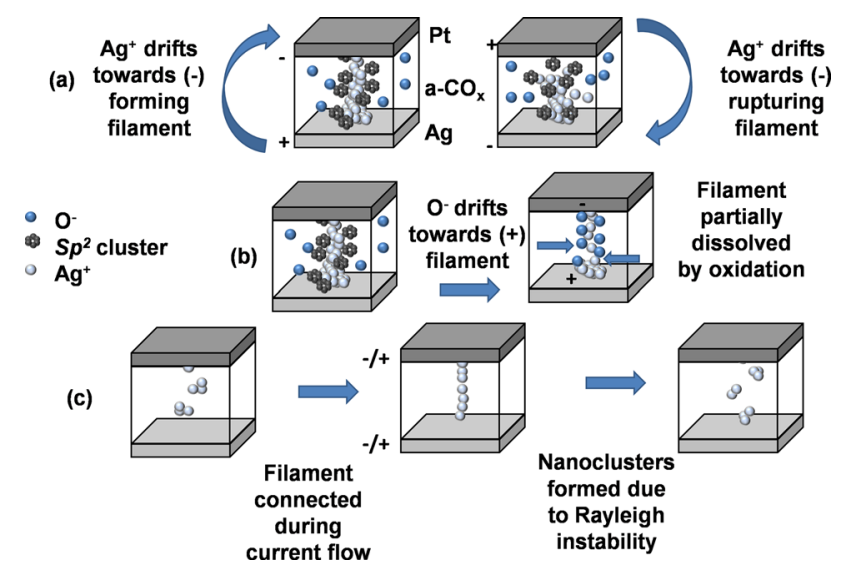

FIG. 6. Illustrations of (a) the non-volatile switching mechanism, (b) the 2nd forming process, and (c) the threshold switching mechanism. 
TABLE I. Summary of the resistive switching characteristics of the $\mathrm{Ag} / \mathrm{a}-\mathrm{CO}_{\mathrm{x}} / \mathrm{Pt}$ devices.

\begin{tabular}{lccccc}
\hline \hline Switching characteristic & Forming mechanism & Ratio $\mathrm{R}_{\mathrm{ON} / \mathrm{OFF}}$ & $\mathrm{V}_{\mathrm{SET}}(\mathrm{V})$ & $\mathrm{V}_{\mathrm{RESET}}(\mathrm{V})$ & Weibull slope $(\beta)$ \\
\hline Non-volatile & Joule heating/metallisation & $\sim 5$ & $1.0 \pm 0.2$ & $-1.65 \pm 0.05$ & $4.5\left(\mathrm{~V}_{\mathrm{SET}}\right) / 24.4\left(\mathrm{~V}_{\mathrm{RESET}}\right)$ \\
Volatile & Filament oxidation & $>10^{5}$ & $1.0 \pm 0.4$ & $<0.2 \mathrm{~V}$ & 2.7 \\
\hline \hline
\end{tabular}

electric field. However, during the transition to volatile threshold voltage switching, the conductance falls to below $2 e^{2} / h$ in both the LRS and HRS. This is suggestive of a discontinuous CF. Previous reports on a-C ECM devices ${ }^{11}$ have highlighted two regions of distinctly different resistive switching characteristics-non-volatile resistive switching (for HRS $>2 e^{2} / h$ ) and volatile threshold voltage switching (for HRS $\ll 2 e^{2} / h$ ). In these devices, the LRS was controlled by the compliance current and as this is reduced, a clear transition takes place from non-volatile to volatile resistive switching behaviour. Our devices exhibit these distinctly different switching characteristics. Their threshold switching can be described by the Rayleigh instability model ${ }^{11,31}$ since the small diameter metallic CF relaxes by diffusion to form disconnected Ag nanoparticles in order to reduce surface energy [Fig. 6(c)]. This behaviour has also been observed by several groups in metal oxides ${ }^{13,18,32}$ and in a-C ECMs. ${ }^{11}$ The stable bi-directional operation of the lower-current threshold switching behaviour at voltages close to the $1 \mathrm{st}$ forming voltage suggests that the devices are less susceptible to Joule heating-induced clustering of $s p^{2}$ bonds, as this may cause the device to revert back to non-volatile switching behaviour. In the limit, where a-C spans the thickness of the device, if a thermal conductivity of $1 \mathrm{~W} \mathrm{~m}^{-1} \mathrm{~K}^{-1}$ is assumed the maximum temperature rise during threshold switching is $225 \mathrm{~K}$ according to Eq. (2). However, the thermal conductivity about the filament is likely to be higher due to the embedded Ag, and thus, the device remains relatively cool. A summary of the characteristics and mechanisms associated with the device operation is shown in Table I.

In summary, the resistive switching mechanisms of $\mathrm{Ag} /$ a- $\mathrm{CO}_{\mathrm{x}} / \mathrm{Pt}$ devices have been investigated. Both volatile and non-volatile memory behaviours were observed. We have demonstrated that this behaviour can be controlled by the polarity and the sequence of the application of bias. The non-volatile switching behaviour was well described by an ECM metallic conducting filament model. We have introduced a model that explains volatile threshold switching behaviour by the reduction of the CFs by oxidation, making them susceptible to dissolution due to Rayleigh instability. The volatile threshold voltage switching characteristics are comparable to those observed in most reported metal oxide devices. ${ }^{32}$ The present work demonstrates the potential for oxygenated amorphous carbon ECM devices to be incorporated into the next generation of neuromorphic computing and memory storage technologies.

The authors gratefully acknowledge the Australian Research Council for financial support (Discovery Project No. DP170102086) and thank Dr. Matthew Field and Dr. Edwin Mayes from the RMIT microscopy and microanalysis facility for assisting with the TEM experiments. This work was performed in part at the Australian National Fabrication Facility (ANFF), a company established under the National
Collaborative Research Infrastructure Strategy, through the La Trobe University Centre for Materials and Surface Science.

${ }^{1}$ H. S. P. Wong and S. Salahuddin, Nat. Nanotechnol. 10, 191 (2015).

${ }^{2}$ M. Di Ventra and Y. V. Pershin, Nat. Phys. 9, 200 (2013).

${ }^{3} \mathrm{~A}$. Chen, paper presented at the 2014 IEEE International Conference on IC Design \& Technology, 2014.

${ }^{4}$ D. Fu, D. Xie, T. Feng, C. Zhang, J. Niu, H. Qian, and L. Liu, IEEE Electron Device Lett. 32(6), 803 (2011).

${ }^{5}$ J. Xu, D. Xie, T. Feng, C. Zhang, X. Zhang, P. Peng, D. Fu, H. Qian, T.-1. Ren, and L. Liu, Carbon 75, 255 (2014).

${ }^{6}$ F. Kreupl, R. Bruchhaus, P. Majewski, J. B. Philipp, R. Symanczyk, T. Happ, C. Arndt, M. Vogt, R. Zimmermann, and A. Buerke, paper presented at the Electron Devices Meeting, 2008, IEDM 2008, IEEE International, 2008.

${ }^{7}$ C. A. Santini, A. Sebastian, C. Marchiori, V. P. Jonnalagadda, L. Dellmann, W. W. Koelmans, M. D. Rossell, C. P. Rossel, and E. Eleftheriou, Nat. Commun. 6, 8600 (2015).

${ }^{8}$ T. Bachmann, W. Koelmans, V. Jonnalagadda, M. Le Gallo, C. Santini, A. Sebastian, E. Eleftheriou, M. Craciun, and C. Wright, Nanotechnology 29(3), 035201 (2018)

${ }^{9}$ Y.-J. Chen, K.-C. Chang, T.-C. Chang, H.-L. Chen, T.-F. Young, T.-M. Tsai, R. Zhang, T.-J. Chu, J.-F. Ciou, and J.-C. Lou, IEEE Electron Device Lett. 35(10), 1016 (2014).

${ }^{10}$ F. Zhuge, W. Dai, C. He, A. Wang, Y. Liu, M. Li, Y. Wu, P. Cui, and R.-W. Li, Appl. Phys. Lett. 96(16), 163505 (2010).

${ }^{11}$ X. Zhao, H. Xu, Z. Wang, L. Zhang, J. Ma, and Y. Liu, Carbon 91, 38 (2015).

${ }^{12}$ X. Zhao, H. Xu, Z. Wang, Z. Xu, C. Zhang, G. Wang, W. Liu, J. Ma, and Y. Liu, J. Mater. Chem. C 5(22), 5420 (2017).

${ }^{13}$ Z. Wang, S. Joshi, S. E. Savel'ev, H. Jiang, R. Midya, P. Lin, M. Hu, N. Ge, J. P. Strachan, Z. Li, Q. Wu, M. Barnell, G.-L. Li, H. L. Xin, R. S. Williams, Q. Xia, and J. J. Yang, Nat. Mater. 16, 101 (2017).

${ }^{14}$ J. Song, J. Woo, A. Prakash, D. Lee, and H. Hwang, IEEE Electron Device Lett. 36(7), 681 (2015).

${ }^{15}$ H. Kröger, C. Ronning, H. Hofsäss, P. Neumaier, A. Bergmaier, L. Görgens, and G. Dollinger, Diamond Relat. Mater. 12(10), 2042 (2003).

${ }^{16}$ F. Kreupl, preprint arXiv:1408.4600 (2014).

${ }^{17}$ W. Weibull, J. Appl. Mech. 18(3), 293 (1951).

${ }^{18}$ S. Tappertzhofen and S. Hofmann, Nanoscale 9(44), 17494 (2017).

${ }^{19}$ J. Frenkel, Phys. Rev. 54(8), 647 (1938).

${ }^{20}$ A. Sebastian, A. Pauza, C. Rossel, R. M. Shelby, A. F. Rodríguez, H. Pozidis, and E. Eleftheriou, New J. Phys. 13(1), 013020 (2011).

${ }^{21}$ Z. Cheng, L. Liu, S. Xu, M. Lu, and X. Wang, Sci. Rep. 5, 10718 (2015).

${ }^{22}$ U. Russo, D. Ielmini, C. Cagli, and A. L. Lacaita, IEEE Trans. Electron Devices 56(2), 186 (2009).

${ }^{23}$ T. Tsuruoka, K. Terabe, T. Hasegawa, and M. Aono, Nanotechnology 22(25), 254013 (2011).

${ }^{24}$ A. A. Balandin, M. Shamsa, W. L. Liu, C. Casiraghi, and A. C. Ferrari, Appl. Phys. Lett. 93(4), 043115 (2008).

${ }^{25}$ A. J. Bullen, K. E. O'Hara, D. G. Cahill, O. Monteiro, and A. Von Keudell, J. Appl. Phys. 88(11), 6317 (2000).

${ }^{26}$ F. Tuinstra and J. L. Koenig, J. Chem. Phys. 53(3), 1126 (1970).

${ }^{27}$ D. D'Angelo, C. Bongiorno, M. Amato, I. Deretzis, A. La Magna, E. Fazio, and S. Scalese, J. Phys. Chem. C 121(9), 5408 (2017).

${ }^{28}$ D. G. McCulloch and A. R. Merchant, Thin Solid Films 290-291, 99 (1996).

${ }^{29}$ K. M. Kim and C. S. Hwang, Appl. Phys. Lett. 94(12), 122109 (2009).

${ }^{30}$ D.-H. Kwon, K. M. Kim, J. H. Jang, J. M. Jeon, M. H. Lee, G. H. Kim, X.-S. Li, G.-S. Park, B. Lee, S. Han, M. Kim, and C. S. Hwang, Nat. Nanotechnol. 5, 148 (2010).

${ }^{31}$ C.-P. Hsiung, H.-W. Liao, J.-Y. Gan, T.-B. Wu, J.-C. Hwang, F. Chen, and M.-J. Tsai, ACS Nano 4(9), 5414 (2010).

${ }^{32}$ W. Zhongrui, R. Mingyi, M. Rivu, J. Saumil, J. Hao, L. Peng, S. Wenhao, A. Shiva, Z. Ye, L. Can, W. Huaqiang, X. Qiangfei, and Y. J. Joshua, Adv. Funct. Mater. 28(6), 1704862 (2018). 